\title{
Relationships between Actin Regulatory Mechanisms and Measurable State Variables
}

\author{
Michael Bindschadler and James L. McGrath \\ Department of Biomedical Engineering, University of Rochester, 601 Elmwood Ave, P.O. Box 639 Rochester, NY, 14642, USA
}

(Received 21 September 2006; accepted 25 January 2007; published online 23 March 2007)

\begin{abstract}
In this report we extend our recent mathematical formulation of the actin cycle model [Bindschadler et al. Biophys. J. 86 (2004) 2720] to predict the influence of key regulatory mechanisms on network-scale state variables estimable in live cell experiments. Specifically, we examine the influence of regulation by cofilin, profilin, capping protein and proteins that adjust filament number through nucleation and/or filament severing, on the higher order variables of average filament length, polymer fraction, and filament turnover rate. Importantly, we find that severing/ nucleation, the acceleration of ADP-subunit disassembly by cofilin, and the catalytic and shuttle functions of profilin have 'signature' effects on the higher order state variables. In this way, measurement of the state variables in live cells can allow inference of regulatory mechanism(s) underlying changes in cell state. Our results compare favorably to published data for endothelial cells undergoing a transition from non-motile confluent cells to highly motile subconfluent cells. The extension of our model to higher order state variables allows us to investigate other important issues such as the distinction between basic and higher order measures of filament dynamics, the influence of thymosin $\beta 4$ on network state variables, the interplay between thymosin $\beta 4$ and profilin, and the synergystic effects of cofilin and profilin.
\end{abstract}

Keywords-Cell motility, Mechanistic modeling, Systems biology, Actin cycle, Cofilin, Profilin, Capping protein, Filament severing, Actin nucleation, Thymosin.

\section{INTRODUCTION}

While actin is a key structural protein in nearly every mammalian cell, its structural functions are inextricably linked to its dynamic nature. The conditions of cytoplasm favor the polymerization of actin monomers (G-actin) into structurally polarized filaments (F-actin) and these are organized into bundles and networks. Assembly of networks at the periphery

Address correspondence to James L. McGrath, Department of Biomedical Engineering, University of Rochester, 601 Elmwood Ave, P.O. Box 639, Rochester, NY 14642, USA. Electronic mail: jmcgrath@bme.rochester.edu of cells is necessary to extend the plasma membrane during cell crawling or spreading, and the assembly of bundles at adhesive contacts is needed to generate intracellular tension.

Because the monomers required for assembly must derive from the disassembly of older filaments, all actin-based structures are continuously recycled in cells. The full array of cellular actin dynamics includes not only steady assembly and disassembly, but also convective flows, contractions and expansions, and both filament and monomer diffusion. These complex dynamics derive from regulation of a cycle of polymerization and depolymerization intrinsic to actin alone. In solutions of pure actin, individual filaments assemble ATP-bound monomers at their 'barbed' end and disassemble ADP-bound monomers at their opposite 'pointed' ends. ${ }^{56}$ The intrafilament flux that results from this imbalance is sustained by ATP hydrolysis within the filament.

Among the many actin binding proteins that have been discovered is a subset of widely expressed proteins that target key steps in actin's intrinsic cycle. ${ }^{44}$ One of the proteins modulating the intrinsic actin cycle is profilin, a monomer binding protein that catalyzes the rate exchange of ADP for ATP on monomeric actin ${ }^{41,49}$ and then permits the assembly of its bound monomer at barbed but not pointed ends. ${ }^{46}$ Because profilin quickly releases from barbed ends, it does not hinder further assembly. ${ }^{19}$ In this way proflin creates a barbed end specific, but otherwise exact, analogue of ATP actin to drive filament assembly. Equally dedicated, but to the destruction of actin filaments is the $\mathrm{ADF} /$ cofilin family of proteins (herein referred to as cofilin). These proteins bind the ADP-bound subunits of filaments found most prominently near pointed ends. ${ }^{9}$ Cofilins destablize filament structure leading to both filament severing ${ }^{31}$ and enhanced depolymerization. ${ }^{9}$

Also regulating the actin cycle in cells are proteins that cap the barbed and pointed ends. Barbed end specific 'capping protein' blocks assembly and 
disassembly at barbed ends. ${ }^{24}$ Tropomyosin and Arp2/ 3 complex can have similar effects at pointed ends but Arp $2 / 3$ complex is most notable for its ability to nucleate new actin filaments. ${ }^{38}$ It has recently become understood that formins represent another family of potent actin nucleators. ${ }^{28}$ While not interacting with filaments directly, thymosins bind actin monomers to help maintain roughly equal amounts of polymerized and unpolymerized actin in cells. ${ }^{48}$

To visualize the dynamics of actin in cells, investigators follow the motions of fluorescent actin tracers introduced by microinjection or ectopic expression. Traditionally, investigators have used fluorescence recovery after photobleaching (FRAP) or photoactivation of fluorescence (PAF) experiments to locally perturb fluorescence. ${ }^{29,53}$ The evolution of fluorescence in these assays reveals the turnover of actin networks, the diffusion of a highly mobile (assumed monomeric) actin species, and the degree of partitioning between these two pools. ${ }^{52}$ Unlike FRAP and PAF, which benefit from high concentrations of fluorescent tracers for strong signals, the more recent technique of fluorescence speckle microscopy (FSM) visualizes actin dynamics by using sensitive cameras to image the movements of individual fluorescent tracers. ${ }^{55}$ This technique has the advantage of a high spatial resolution that allows the construction of maps of actin dynamics, and analysis can separate network convection and filament turnover in these maps. ${ }^{54}$ Fluorescent actin in the highly mobile population moves too fast to be detected, and so the technique cannot measure monomer diffusion or the fraction of actin polymerized.

While turnover rates and polymer fractions are estimable in live cell experiments, these quantities are complex manifestations of the more fundamental concentrations and activities of actin and its regulatory proteins. The lengths of filaments in cellular networks are also complex functions of actin cycle regulation. These lengths can be inferred by electron microscopy, ${ }^{51}$ and in assays that count filaments using permeabilized cells to nucleate exogenous actin. ${ }^{8}$ Thus the filament turnover, the polymer fraction, and average filament lengths are estimable parameters that carry information about the state of the actin cycle. For the purposes of this report we will reserve the term 'state variables' as reference to these three parameters.

The purpose of the current effort is to connect the state variables to our recently published mechanistic model of the actin cycle. ${ }^{3}$ The model predicts species concentrations and fluxes as a consequence of basic rates and activities of actin and actin binding proteins. Specifically the model represents the activities of capping protein, profilin, cofilin, and other proteins that control the number of filaments through severing and/ or nucleation. Most significant for interpreting cellular experiments, we ask if key modes of regulation affect the state variables in ways that could be considered 'signatures.' If so, then examining changes in the state variables could identify the mechanism underlying changes in cytoskeletal dynamics.

Our results do suggest mechanistic signatures for the activities of cofilin, profilin, and for regulatory proteins that change filament number through nucleation and severing. The model predictions of state variable changes compare favorably to earlier experiments in which we both measured the state variables and identified regulatory mechanisms through biochemical analysis in endothelial cells. ${ }^{34}$ As a system of homogenous, steady state equations, the published model must be advanced to respect the complexity of cells. The current results encourage the development of a temporally and spatially resolved mechanistic model as a tool for analyzing cellular experiments.

\section{STATE VARIABLES}

The three state variables of interest here are the polymer fraction $P F$, turnover rate $T R$, and average filament length $L_{\text {avg. }}$. These quantities are chosen because they: (1) describe the state of the actin cycle at the network level, (2) are estimable in cells, and (3) can be related to the published model's variables. In this section we define each of these quantities in detail and discuss how they can be measured in cells and how they can be related to variables already included in the actin cycle model.

\section{Polymer Fraction}

The polymer fraction is the fraction of total cellular actin in filamentous form. Biochemically, it can be measured by comparing the quantity of actin that resists triton extraction to total cellular actin. ${ }^{6}$ The fluorescence-based methods of FRAP and PAF can also be used to estimate the polymer fraction. In FRAP, fluorescently derivatized actin is introduced to cells either through microinjection or expression, and a laser is used to rapidly photobleach a region of the cell. ${ }^{29}$ The rate of return of fluorescence to the bleached region is monitored and can be separated into a fast phase (the diffusion of monomers) and a slow phase (the turnover of filaments). ${ }^{52}$ The relative fluorescence contributions of these two phases indicate the relative amounts of monomeric and polymeric actin. In PAF, the introduced actin has its fluorophore chemically "caged" with a photolabile bond so that it only becomes fluorescent when exposed to light. ${ }^{53}$ As with FRAP, a region of a cell is briefly exposed to laser light 
(uncaging the fluorophore in that region), but the effect is to light up the region rather than bleaching it. In PAF, it is the decay of fluorescence in the photoactivated region which is observed, but the interpretation of the dynamics is identical; there is a fast phase attributed to monomer diffusion and a slow phase attributed to filament turnover. These fluorescencebased methods have the advantage over the biochemical measurements in that they are made in living, intact cells.

\section{Turnover Rate}

The turnover rate is the number of a filament's component subunits that are renewed per unit time, or more simply, the reciprocal of a subunit's residence time in a filament. Actin subunits typically assemble at the barbed end of a filament, flux through to the pointed end, and disassemble. Both FRAP and PAF can provide estimates of the filament lifetime in living cells because the time required for recovery (or decay) reflects the time for replacement of all subunits present at the moment of fluorescence perturbation. The newer technique of FSM can also measure turnover rate ${ }^{55}$ because the time between speckle appearance and disappearance corresponds to the transit time of subunits through a filament. With FSM it is possible to construct maps of local actin turnover in different parts of a cell, as well as additional properties such as network convection. ${ }^{54}$

\section{Average Filament Length}

The average filament length can be defined as the concentration of polymerized actin divided by the concentration of filaments, however the concentration of filaments actin networks is difficult to measure. Several groups ${ }^{8,21,34}$ have estimated the number of filaments in cells according to the following procedure: (1) Cells are partially extracted by washing with weak detergent, then exposed to pyrene actin (a fluorescent actin which is $20 \times$ brighter when polymerized ${ }^{27}$ and cytochalasin B (a fungal poison which irreversibly blocks barbed ends of filaments ${ }^{7}$ ); (2) The rate of increase in fluorescence allows determination of how rapidly the new actin polymerizes onto filament pointed ends; and (3) this rate is divided by the rate at which actin polymerizes onto a single pointed end to give an estimate of the number of free pointed ends. ${ }^{21}$ Combined with an estimate of the fraction of capped pointed ends (the cited groups assumed all pointed ends are free), and a geometric estimate of cell volume, one can calculate an estimate of the filament concentration. While this method is highly indirect, it is quantitative. Unfortunately, the density of interwoven filaments in the cytoskeleton precludes an accurate measurement of average filament lengths from electron micrographs.

\section{Relation of State Variables to Actin Cycle Variables}

The three state variables can be related to existing model variables through these simple equations

$$
\begin{gathered}
L_{\mathrm{avg}}=\frac{f}{n} \\
P F=\frac{f}{A_{\mathrm{tot}}} \\
T R=\frac{q}{L_{\mathrm{avg}}}
\end{gathered}
$$

where $f$ is the concentration of polymerized actin, $n$ is the concentration of filaments, $A_{\text {tot }}$ is the total concentration of actin, and $\vec{q}$ is the flux from barbed to pointed end.

\section{METHODS}

\section{Mathematical Model}

We use the published actin cycle model ${ }^{3}$ plus Eqs. (1-3) above for all calculations. The 30 non-linear algebraic rate equations and key tables of parameter values are included in the Appendix A. Note that the model does not explicitly represent any proteins except for thymosin $\beta 4$ and profilin. Instead the key activities of most regulatory proteins are treated as separable. For example, the multiple functions ascribed to ADF/ cofilin: severing, accelerated disassembly, and enhanced $P i$ release on filaments, are individually controllable in the model to avoid a complex, unjustified assumption about how they might jointly depend on ADF/cofilin concentration. The model and its solvers are available at http://mcgrathlab.urmc.rochester.edu/ActinCycle/. In fact, all of the model data presented in this paper were gathered over the web using only a web browser.

We note that all the assumptions and simplifications of Bindschadler et al. are present here as well. Significant among are these are the need to treat all filaments as equal in length and severing as indiscriminant. While some severing mechanisms (ex. gelsolin) are thought to be indiscriminant, other ADF/cofilin will only sever filaments at ADP bound spans where it binds.

\section{Parameter Choices}

The concentration of total actin $(100 \mu \mathrm{M})$ and the range on filament concentration $(0.1 \mu \mathrm{M}-0.6 \mu \mathrm{M})$ are 
estimates from experiments on endothelial cells. ${ }^{34}$ This range is presumably regulated by a combination of severing and nucleation but our model is currently unable to distinguish between these possibilities. We also underscore that while the Arp2/3 complex provides one mechanism for increasing filament number through nucleation, we do not explicitly model the Arp2/3 complex here because we cannot yet tie pointed end capping activity to nucleation rates until the data become available. We did examine the isolated effects of increased pointed end capping on state variables and found a monotonic decrease in turnover, and monotonic increases in polymer fraction and filament length. These results are not included.

Thymosin $\beta 4$ was assumed equimolar with actin, consistent with its concentrations in most cells $^{12,20,39,44,47}$ and with its function as the major sequestering protein. ${ }^{23,48}$ Measurements of the concentration of profilin in cells range from $5 \mu \mathrm{M}$ in chick brain $^{12}$ to $55 \mu \mathrm{M}$ in platelets. ${ }^{17}$ However, not all profilin is necessarily available for binding to actin, as profilin has over 50 identified binding partners, ${ }^{58}$ and in particular, it has been demonstrated that binding to phosphoinositides dissociates profiling-actin complexes. ${ }^{30}$ Here we vary the effective concentration of profilin from $0 \mu \mathrm{M}$ to $20 \mu \mathrm{M}$ to simulate regulation.

The range of rates for ADP-actin subunit depolymerization from pointed ends $\left(k_{P D}^{-}\right)$and the rate of inorganic phosphate $(\mathrm{Pi})$ release from ADP.pi-actin subunits in filaments, $\left(k_{f D \cdot P i \rightarrow D}^{-}\right)$, have not been measured in cells, so we use rates from in vitro experiments. The only known influences on these rates are proteins of the ADF/cofilin family, which are present at significant concentrations in cells, $\sim 20 \mu \mathrm{M} .{ }^{1,12,14,44}$ Cofilins bind preferentially to ADP-bound subunits in actin filaments and accelerate depolymerization of these filaments by increasing the off-rate of ADP-actin subunits, ${ }^{9}$ severing filaments to create more ends, ${ }^{31}$ and increasing the $\mathrm{Pi}$ release rate on ADP.Pi-bound subunits. ${ }^{5}$ However, it is not clear what the relative contributions of these three effects are and how they relate to the concentration of cofilin. Carlier et al., ${ }^{9}$ assuming the effect is entirely due to a shift in $k_{P D}^{-}$, calculated that cofilin increases depolymerization 25fold (and later 50 -fold ${ }^{13}$ ), but this is surely an overestimate. On the other hand, Moriyama, ${ }^{37}$ by simultaneously accounting for severing and $k_{P D}^{-}$increases, found an acceleration of only 6.4 -fold. This is likely an underestimate since only a low concentration of cofilin was used $(0.8 \mu \mathrm{M})$ due to methodological constraints, and since cofilin binding is cooperative. ${ }^{32}$ Finally, because cofilin binding to actin is inhibited by phosphorylation, knowledge of $k_{P D}^{-}$, the concentration of cofilin, and how $k_{P D}^{-}$and $k_{f D \cdot P i \rightarrow D}^{-}$depend on the concentration of active cofilin, would still not be suffi- cient to fully determine kinetics in cells. For the present work, we allow $k_{P D}^{-}$to increase 20 -fold and to $k_{f D \cdot P i \rightarrow D}^{-}$ increase 15 -fold ${ }^{5}$ from their values for pure actin. We vary $n$ independently of cofilin parameters since many other proteins affect the overall balance between severing and nucleation, but when we consider cofilin alone, we allow it to double the filament concentration.

\section{RESULTS}

We begin our analysis by illustrating the important distinctions between the state variable $T R$ and the rates of subunit flux and ATP hydrolysis, three quantities that have often been confused in the literature. We then move to an investigation of the effects of individual regulated mechanisms on state variables, both with and without thymosin $\beta 4$. We examine more closely the interaction between thymosin $\beta 4$ and profilin and between profilin and cofilin, and finally, compare model predictions with measurements in living endothelial cells.

\section{Filament Turnover, Subunit Flux, and ATP Hydrolysis are Distinguishable Quantities}

In quantifying how dynamic actin filaments are, investigators have often used the rate of ATP hydrolysis as an accessible measure in vitro ${ }^{9,13}$ and filament turnover in vivo ${ }^{29,34,53}$. Both of these quantities are often conceptually equated with the filament flux, when in fact all three measures can behave independently. While ATP hydrolysis is required to drive flux, not all the energy from hydrolysis is used productively, and so the flux rate and the rate of ATP hydrolysis are not equal in general. ATP hydrolysis and turnover are also independent quantities because only the latter depends on filament length; at a given flux shorter filaments 'turnover' faster than longer filaments.

To demonstrate the independence of flux from ATP hydrolysis and turnover, we vary the number of filaments in a simulated solution of actin filaments at steady state. As the number of filaments increases, the flux rate falls while the turnover and ATP hydrolysis rates rise (Fig. 1A). The increase in the number of filament ends (ATPases) increases the hydrolysis rate but reduces the monomer pool of ATP-actin. This reduces net assembly at barbed ends and thereby reduces flux. The turnover rate rises despite the falling flux because the dramatic shortening of filaments (the same amount of actin is spread over a greater number of filaments, reducing average length) more than compensates for the reduction in flux. The independence of hydrolysis from turnover and flux can be appreciated by reducing the fraction of free pointed ends from 1 to 0 (Fig. 1c). 

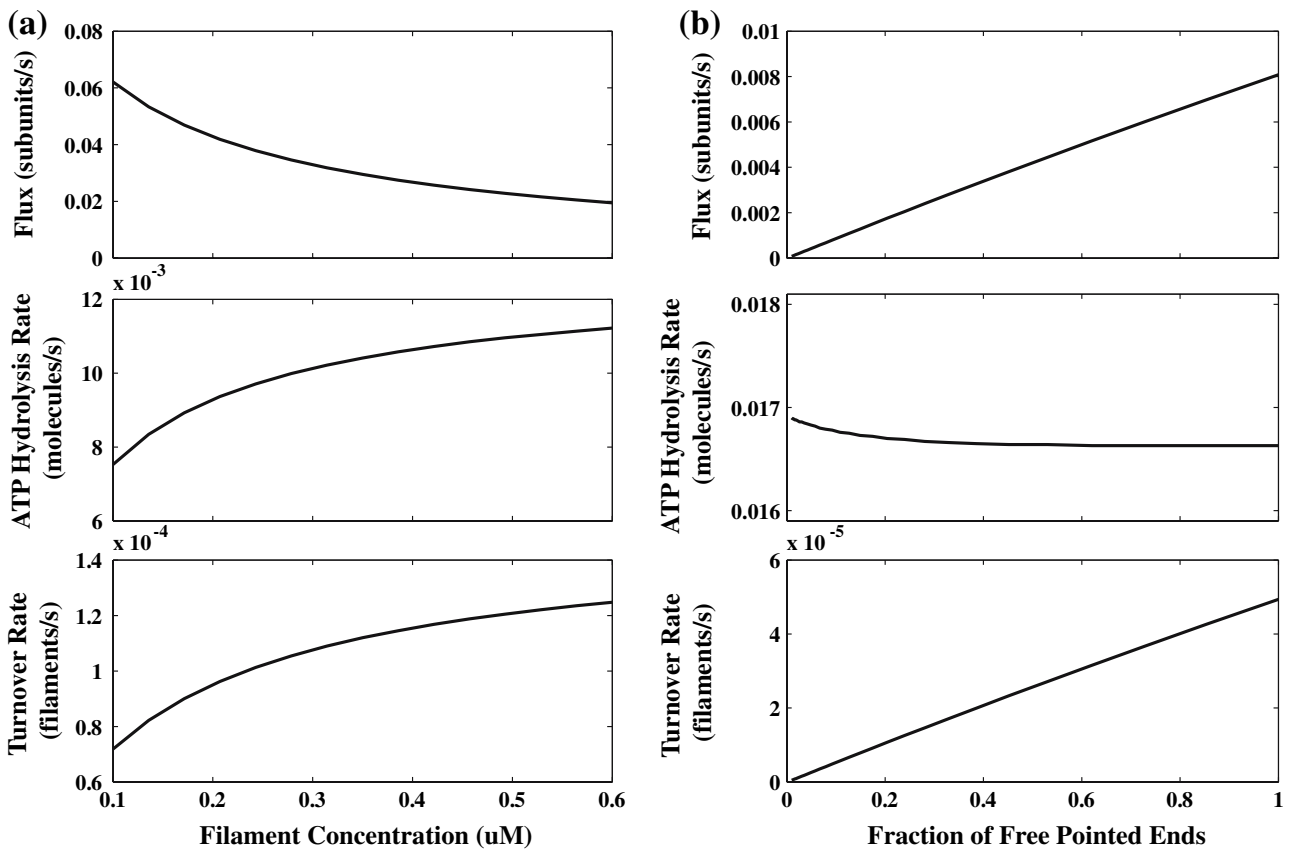

FIGURE 1. Flux, hydrolysis rate and turnover are not redundant measurements (a) Increasing filament concentration $(n=0.1--$ $0.6 \mathrm{nM}$ ) increases hydrolysis, but decreases turnover rate and flux. (b) Capping pointed ends ( $\beta=1$ to 0.01 , read from right to left) reduces flux and turnover, but hydrolysis is essentially unaffected. Unvaried parameters are held at rest values.

Without free pointed ends, flux and turnover reduce to zero while non-productive ATP hydrolysis continues at barbed ends. In this limit, ATP-actin monomers assemble, hydrolyze, and disassemble, all at the barbed end. Thus, while intimately related, it is important to keep in mind the distinctions between these three measures of actin dynamics.

\section{Effects of Individual Mechanisms on State Variables}

We now investigate the influence of actin cycle regulation on the state variables. We define a "rest" value and an "activated" value for each mechanism (Table 1), based on measurements from endothelial cells (for $n$ and $\alpha$ ) or ranges from the literature $\left(k_{f D \cdot P i \rightarrow D}^{-}, k_{P D}^{-}\right.$, and $\left.P_{\text {tot }}\right)$. The direction of mechanism change representing activation was guided by experiments on neutrophils and platelets, where stimulation leads to filament uncapping, ${ }^{2,15}$ nucleation, ${ }^{57}$ and cofilin activation. ${ }^{50}$
Turning each mechanism on individually from the rest state (Fig. 2a), we find that each has a distinct effect on the state variables. Accelerated depolymerization (from $A D F /$ cofilin activity) and increased profilin activity are the most potent activators of filament turnover, while the other three mechanisms have lesser effects. The average filament length is decreased dramatically by increasing the number of filaments and significantly by accelerating depolymerization, while being increased by profilin addition (after an initial dip) and by uncapping barbed ends. The polymer fraction is decreased significantly by accelerating depolymerization and slightly by small amounts of profilin, while larger amounts of profilin, barbed end uncapping, and increasing the number of filaments all increase the polymer fraction to over $90 \%$.

Starting instead from the "active" state (all mechanisms activated) and turning each off individually (Fig. 2b) gives a different perspective. All five mechanisms contribute to the high rate of turnover in the

TABLE 1. Measured or estimated ranges for all regulated mechanisms with literature sources.

\begin{tabular}{lllll}
\hline Symbol & \multicolumn{1}{c}{ Description } & \multicolumn{1}{c}{ Rest } & Activated & \multicolumn{1}{c}{ Source } \\
\hline$n$ & Number concentration of filaments & $0.1 \mu \mathrm{M}$ & $0.6 \mu \mathrm{M}$ & McGrath et al. 34 \\
$\alpha$ & Fraction of uncapped barbed ends & 0.05 & 0.30 & McGrath et al. 34 \\
$P_{\text {tot }}$ & Total effective concentration of profilin & $0 \mu \mathrm{M}$ & $20 \mu \mathrm{M}$ & See text \\
$k_{P D}^{-}$ & Rate of ADP-actin subunit depolymerization from pointed ends & $0.3 \mathrm{~s}^{-1}$ & $6 \mathrm{~s}^{-1}$ & See text \\
$k_{f D \cdot P i \rightarrow D}^{-}$ & Rate of inorganic phosphate release from ADP.Pi-actin subunits & $0.0026 \mathrm{~s}^{-1}$ & $0.039 \mathrm{~s}^{-1}$ & Blanchoin and Pollard 5 \\
\hline
\end{tabular}



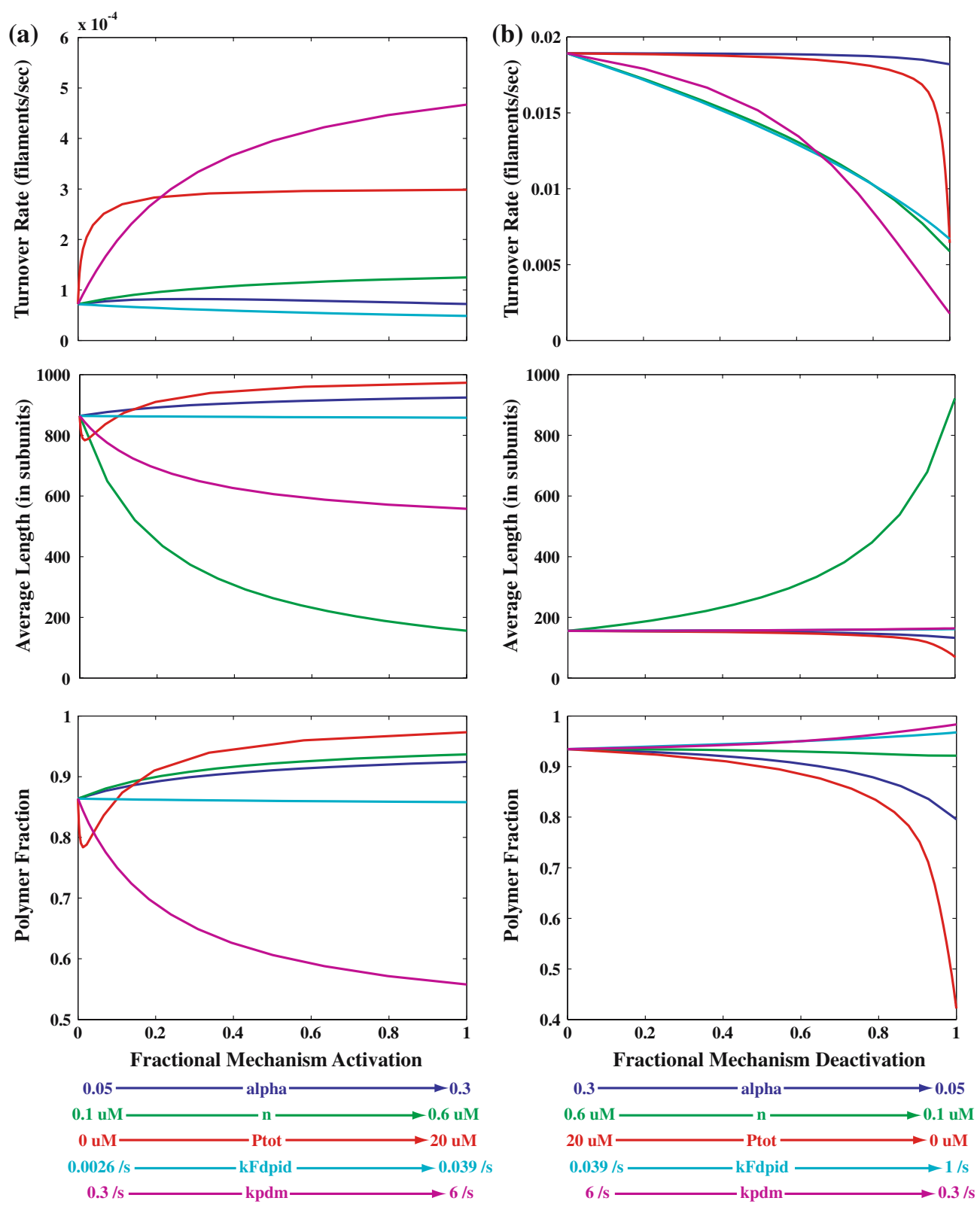

FIGURE 2. The effects of regulated mechanism on state variables. (a) Each mechanism is varied from its rest value to activated value while all others are held at their rest value. (b) Each mechanism is varied from its activated value to its rest value while all others are held at their activated value

"active" state, since turning any one of them off decreases the turnover. For average length, again it is the number of filaments which is the strongest determinant. Reducing filament number increases length over fourfold. Removing profilin more modestly decreases average filament length, reducing it by half. The polymer fraction is high in the active state, and remains high except when nearly all profilin activity is removed or, to a lesser extent, when barbed ends are capped.

A key question is whether these mechanisms have signature effects, which would allow potential identification of underlying mechanisms based only on knowledge of state variable changes. We investigate this in Table 2, and the answer seems to be a qualified yes. If one observes a large change in average filament length, the most likely underlying mechanism is a change in the relative balance of severing/ nucleation of filaments (to produce a change in $n$ ). A large decrease in polymer fraction accompanied by a large increase in turnover is likely due to accelerated depolymerization, while a large decrease in polymer fraction with a large decrease in turnover is more likely due to a large decrease in the activity or quantity of profilin. However, there do not seem to 
TABLE 2. Regulated mechanisms have signature effects.

\begin{tabular}{|c|c|c|c|c|c|c|}
\hline \multirow[t]{2}{*}{ Mechanism Name } & \multicolumn{3}{|c|}{ On from rest } & \multicolumn{3}{|c|}{ Off from active } \\
\hline & TR & Lavg & PF & TR & Lavg & PF \\
\hline$\alpha$ & $\mathbf{x}$ & ++ & ++ & - & $\mathrm{X}$ & --- \\
\hline$n$ & ++ & ---- & ++ & --- & ++++ & $\mathbf{x}$ \\
\hline$P_{\text {tot }}$ & ++++ & ++ & ++++ & --- & -- & ---- \\
\hline$k_{P D}^{-}$ & -- & - & - & --- & $\mathbf{x}$ & + \\
\hline$k_{f D \cdot P i \rightarrow D}^{-}$ & ++++ & --- & ---- & ---- & $x$ & + \\
\hline
\end{tabular}

Markings indicate direction and qualitative magnitude of effect: $(++++)(----)$ Largest/Very Large effect, $(+++)(---)$ Moderate effect $(++)(--)$ Small effect, $(+)(-)$ Very Small effect, X No effect/Essentially no effect. The unique pattern of effects of each mechanism constitutes its signature.

be any state variable changes which could point directly to a change in barbed end capping or the rate of Pi release.

\section{The Influence of Thymosin $\beta 4$}

Pure actin polymerizes until only $\sim 0.1 \mu \mathrm{M}$ of monomeric actin remains, but cells typically maintain pools of unpolymerized actin at hundreds of times this concentration. ${ }^{6}$ This is made possible in many cells by the sequestering protein thymosin $\beta 4$, which binds in a 1:1 complex with monomers and prevents their assembly into filaments. Thymosin $\beta 4$ inhibits nucleotide exchange on bound monomers, but also has a 100-fold higher affinity for ATP-bound monomers than ADP-bound monomers, and therefore generally binds monomers after they have exchanged ADP for ATP. ${ }^{10,25}$ The differences between Fig. 2 (with $100 \mu \mathrm{M}$ thymosin $\beta 4$ ) and Fig. 3 (with no thymosin $\beta 4$ ) illustrate the influence of thymosin $\beta 4$ on state variable values and dynamic ranges.

The most striking effect of thymosin $\beta 4$ is on the polymer fraction. In the absence of thymosin $\beta 4$, the rest polymer fraction is nearly 0.99 and the activation of individual mechanisms maximally reduces it to only 0.94 (Figure 3B, bottom). In contrast, with $100 \mu \mathrm{M}$ thymosin $\beta 4$, the rest polymer fraction is 0.86 and activating $k_{P D}^{-}$reduces it by $30 \%$ to 0.56 (Fig. 2 b, bottom). More subtly, the absence of thymosin $\beta 4$ inverts the small effects of uncapping and severing, changing them from noticeable increases to very small decreases. Deactivating mechanisms individually from the active state, we see that the absence of thymosin $\beta 4$ again both raises the polymer fraction and decreases its dynamic range, but does not change the direction of any changes. Interestingly, without thymosin $\beta 4$, there is no initial drop in polymer fraction at low levels of active profilin. This effect is examined in more detail below.

For average length, altering the balance of severing/ nucleation is the most powerful mechanism with or without thymosin $\beta 4$. Whether being turned on from rest or off from active, the effect of $n$ on average length is essentially independent of thymosin $\beta 4$. The other mechanisms have their dynamic range reduced and altered in a way which mirrors thymosin $\beta 4$ 's effect on polymer fraction, though the effect is much more noticeable when they are being activated from rest than deactivated. Finally, thymosin $\beta 4$ 's main effects on turnover rate are (1) to significantly increase the effectiveness of accelerating depolymerization from the rest state and (2) to slightly increase the effect of capping and profilin removal from the active state.

To summarize, by buffering the ATP-actin monomer pool, thymosin $\beta 4$ lowers the polymer fraction and potentiates the effect of accelerating depolymerization on all three state variables. While the most significant effects of thymosin $\beta 4$ are on the polymer fraction, our study suggests that the protein cannot maintain a large sequestered pool on its own. Instead ADF/cofilin activity must be present to shift the degree of polymerization, and thymosin $\beta 4$ will accentuate this shift so long as profilin is not present. Furthermore, little profilin activity can be present for thymosin $\beta 4$ to maintain a depolymerized actin pool. These results may seem counter the idea that thymosin $\beta 4$ is the major sequestering protein in cells, however the results are a direct consequence of rate constants and mechanisms reported in the literature for thymosin $\beta 4$, actin and profilin. Specifically, the affinity of ATP-monomer for barbed ends is $10 \times$ higher than for thymosin $\beta 4$ and profilin ${ }^{25}$ (see Appendix Table A1). Thus significant numbers of free barbed ends effectively compete monomer away from thymosin $\beta 4$. With profilin's ability to bind ADP monomer, catalyze ADP/ATP exchange, and facilitate monomer assembly at barbed ends, significant amounts of profilin will 'short-circuit' disassembling monomers so that they never see thymosin $\beta 4$. The complex interplay is now explored in more detail by examining the effects of very small amounts of profilin on cycle dynamics when thymosin $\beta 4$ is present.

\section{Profilin and Thymosin $\beta 4$}

The interplay between profilin and thymosin $\beta 4$ has been the subject of some investigation in the litera- 

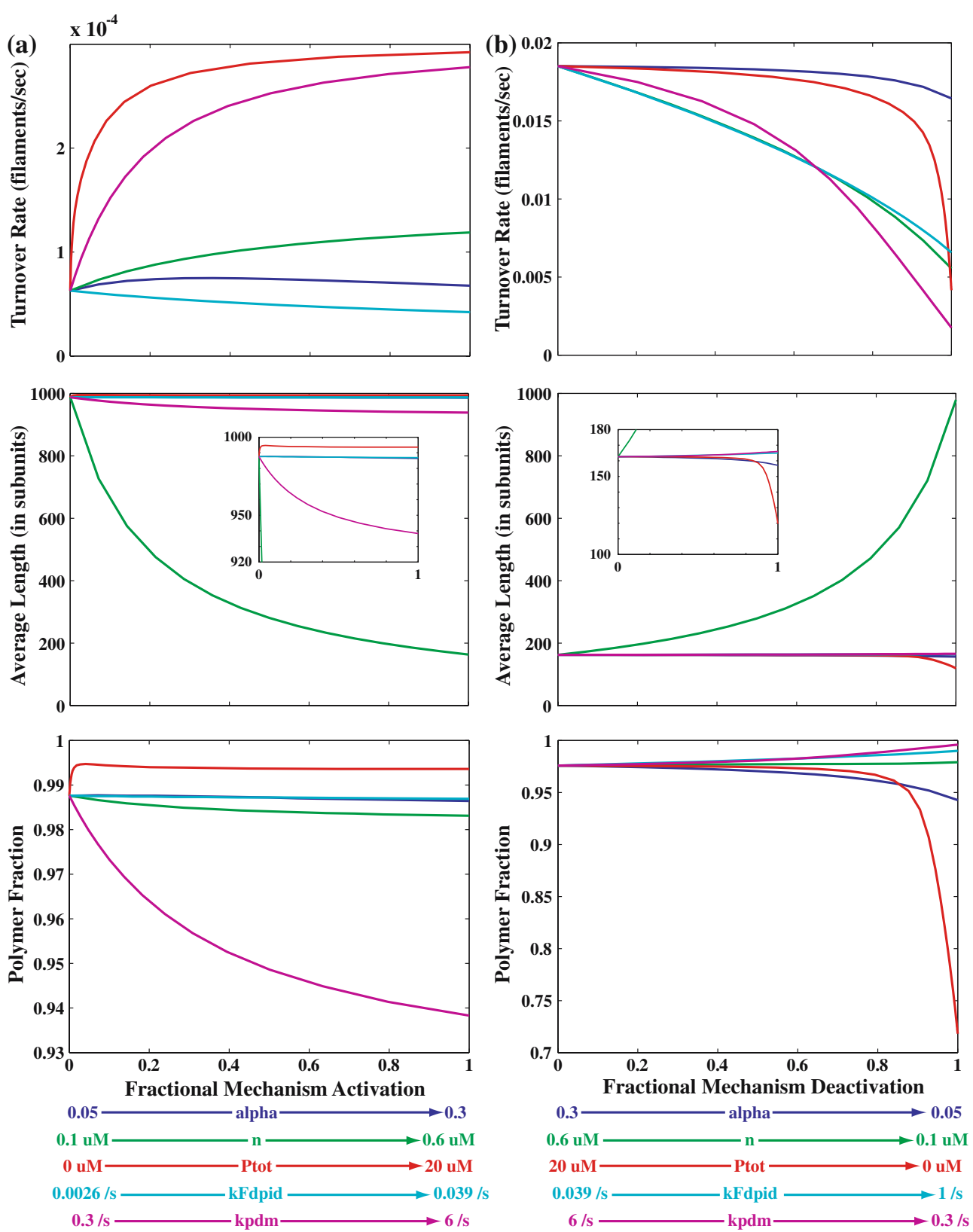

FIGURE 3. Regulation in the absence of thymosin $\beta 4$. (a) Each mechanism is varied from its rest value to activated value while all others are held at their rest value. (b) Each mechanism is varied from its activated value to its rest value while all others are held at their activated value. Insets expand a region of the $y$-axis for clearer viewing. This Figure is identical to Fig. 2 except that thymosin $\beta 4$ is absent in these simulations.

ture. ${ }^{18,41}$ Both proteins bind actin monomers in a $1: 1$ complex with millimolar affinity and change between monomers on a time scale of seconds, but they have opposite effects on nucleotide exchange. thymosin $\beta 4$ strongly inhibits exchange while profilin catalytically accelerates it over 100-fold (see Appendix Table A1). Binding to thymosin $\beta 4$ prevents monomer assembly at both ends of filaments, while profilin prevents assembly at the pointed end but not the barbed end. We noted above that in the presence of thymosin $\beta 4$ a small amount of profilin leads to a decrease in polymer fraction and average length, but more profilin has the opposite effect. This initial dip disappears in the absence of thymosin $\beta 4$, indicating that it is thymosin $\beta 4$-dependent.

To determine the source of the biphasic effect of profilin in the presence of thymosin $\beta 4$, we examine the details of monomer pool compositions and filament end fluxes more closely (Fig. 4). Without profilin activity, most actin is filamentous (86.4\%), and most 
monomeric actin is ATP-bound and sequestered by thymosin $\beta 4$ ( $11.4 \%$ of total actin). Of the remaining $2.2 \%, 1.1 \%$ is free ADP-bound actin monomer, $1 \%$ is ADP-bound monomer sequestered by thymosin $\beta 4$, and $0.1 \%$ is free ATP-bound monomer (Fig. 4a). Adding a small amount of profilin begins to convert the pool of free ADP monomer to free ATP monomer, and the drop in the ADP monomer level leads to increased depolymerization from pointed ends (Fig. 4b). Without thymosin $\beta 4$, this increased depolymerization is more than balanced by increased polymerization of ATP actin (and profilin-ATP actin) at the barbed end so that the net result is increased flux and increased polymer fraction (see Fig. 3, bottom panel). But with thymosin $\beta 4$, the ATP monomer produced is instead sequestered (thymosin $\beta 4$-ATPactin reaching $>20 \mu \mathrm{M}(>20 \%)$. Free barbed ends have a fivefold higher affinity for ATP monomer than thymosin $\beta 4$, but since there are so few of them in the rest state $(0.1 \mu \mathrm{M}$ filaments $\times 0.05$ fraction uncapped $=5 \mathrm{nM}$ free barbed ends), they do not effectively compete with $100 \mu \mathrm{M}$ thymosin $\beta 4$ for the free ATP monomer being created.

As the amount of profilin increases beyond $0.3 \mu \mathrm{M}$ two factors overcome depolymerization. First, ATPactin is produced more and more quickly, and as the concentration rises more ATP-actin finds barbed ends before being absorbed by thymosin $\beta 4$. Second, the concentration of profilin-ATP-actin begins to become significant, and profilin-ATP-actin is protected from being sequestered by thymosin $\beta 4$ but still free to polymerize at the barbed end. When the concentration of profilin reaches $0.3 \mu \mathrm{M}$, the monomer pool not bound to thymosin $\beta 4$ shifts from being mostly ADP-bound to mostly ATP-bound, and this shift occurs at the transition point of all these plots (the

(a)

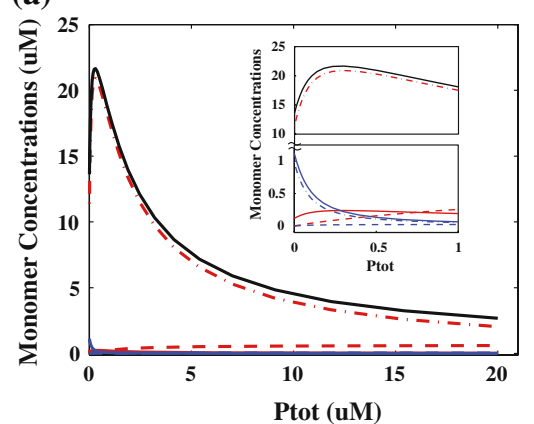

polymer fraction and average length have minima, the thymosin $\beta 4$-ATP-actin concentration reaching a maximum).

In summary, at low concentrations, profilin's catalytic exchange function effectively converts ADP-actin monomers to sequestered ATP-actin-thymosin $\beta 4$ monomers, allowing for more depolymerization from ADP-rich pointed ends. In this phase, profilin's barbed end shuttle function is ineffective because it relies on the chance of a barbed end and a profilin-actin monomer interacting, and both are in extremely short supply. However, at higher concentrations of profilin, the concentration of profilin-actin increases and profilin acts as a shuttle that converts ADP-actin monomers to new ATP-actin subunits on barbed ends. This closes the loop in the actin cycle, and since barbed ends can elongate much more vigorously than pointed ends depolymerize, this ultimately shifts the polymer fraction back upwards. This is the second phase of the biphasic effect. In the absence of thymosin $\beta 4$ to sequester ATP-monomer, profilin's catalytic effect is sufficient to create unsequestered ATP-actin monomers and enhance assembly.

\section{Synergy Between Cofilin and Profilin}

Strong synergy has been reported between the effects of cofilin and profilin in accelerating filament turnover up to 125 -fold. ${ }^{13}$ Both proteins have multiple effects on the actin cycle. Cofilin binds to and destabilizes filaments near their ADP-rich pointed ends, leading to filament severing, acceleration of ADP-actin disassembly, and acceleration of Pi release. Profilin binds to monomeric actin and accelerates nucleotide exchange, recharging monomers for reassembly, and prevents bound monomer from assembling at the

(b)

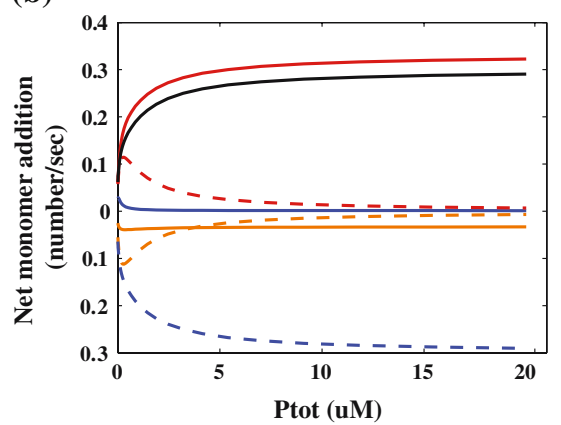

FIGURE 4. Profilin Effects in the Presence of thymosin B4. (a) Effects on Monomer Pool. Main: Shows the steady state concentrations of all monomer species as a function of profilin concentration. Inset: Detail of $0 \mu \mathrm{M}$ to $1 \mu \mathrm{M}$ profilin range. All red curves: ATP-bound actin monomer; All blue curves: ADP-bound actin monomer; Dashed curves: profilin-bound actin monomer; Dash-dot curves: thymosin $\beta 4$ sequestered monomer; Solid curves except black: Uncomplexed actin monomer; Black solid curve: Total concentration of all monomer species. (b) Components of Flux. All curves show net assembly (disassembly if negative) of actin subunits bound to a particular nucleotide. Black solid curve: Total subunit flux through filaments. Other solid curves: Net addition at the barbed end; Dashed curves: Net addition at the pointed end; Red curves: ATP-actin subunits; Blue curves: ADPactin subunits; Orange curves: ADP.Pi-actin subunits. 
pointed end, but allows assembly at the barbed end. It is easy to understand how these activities complement one another: cofilin accelerates the depolymerization of filaments from their pointed ends by increasing the number of pointed ends (through severing), increasing the ADP-actin content of those ends (by accelerating Pi release), and increasing the rate at which ADP-actin disassembles from those ends. Meanwhile, profilin binds newly depolymerized ADP-subunits, recharges them with ATP, and shuttles them to the barbed ends by protecting them from both sequestration by thymosin $\beta 4$ and non-productive reassembly at pointed ends. Thus these two regulatory proteins perform a hand-off that is sufficient to streamline the actin cycle. ${ }^{3}$ We examine the quantitative implications of this conceptual model in Fig. 5.

Since there is not a consensus view of how cofilin's functions relate to one another quantitatively or to the concentration of cofilin, there is not a clear choice regarding what "cofilin activation" should mean in our model. For the purposes of this figure, we consider cofilin activation to be a linear transition across the full range of $k_{P D}^{-}$and $k_{f D \cdot P i \rightarrow D}^{-}$values, and a doubling of the number of filaments from $0.1 \mu \mathrm{M}$ to $0.2 \mu \mathrm{M}$ (to represent severing activity).

With these assumptions, we find that our model also predicts cofilin and profilin to be powerfully synergistic in enhancing filament turnover (Fig. 5). Profilin alone increases turnover rate just over four-fold, while cofilin alone increases it 7.5-fold, but together they increase the turnover rate an incredible 127-fold. Perhaps not surprisingly since all three of cofilin's mechanisms reduce average length and polymer fraction, cofilin activation strongly reduces these state variables also. Profilin has the opposite effect on both. The effects of combining cofilin and profilin on average length are dominated by the contribution of cofilin, with profilin moderating the effect slightly. For the polymer fraction, however, their contributions are more balanced, dipping initially but then stabilizing and remaining approximately constant beyond $5 \%$ activation.

These last two results highlight the fact that it is possible for mechanisms to mask one another's effects. Profilin activation is associated with increased average length, but the combination of cofilin and profilin activity shows significantly reduced average length, and this masks the signature of profilin. Similarly, the combination of cofilin and profilin activation stabilizes the polymer fraction, masking cofilin's signature effect of decreasing polymer fraction.

\section{Comparison to Endothelial Cells}

McGrath et al. ${ }^{34}$ measured actin filament length, number, turnover rate, barbed end exposure, cofilin
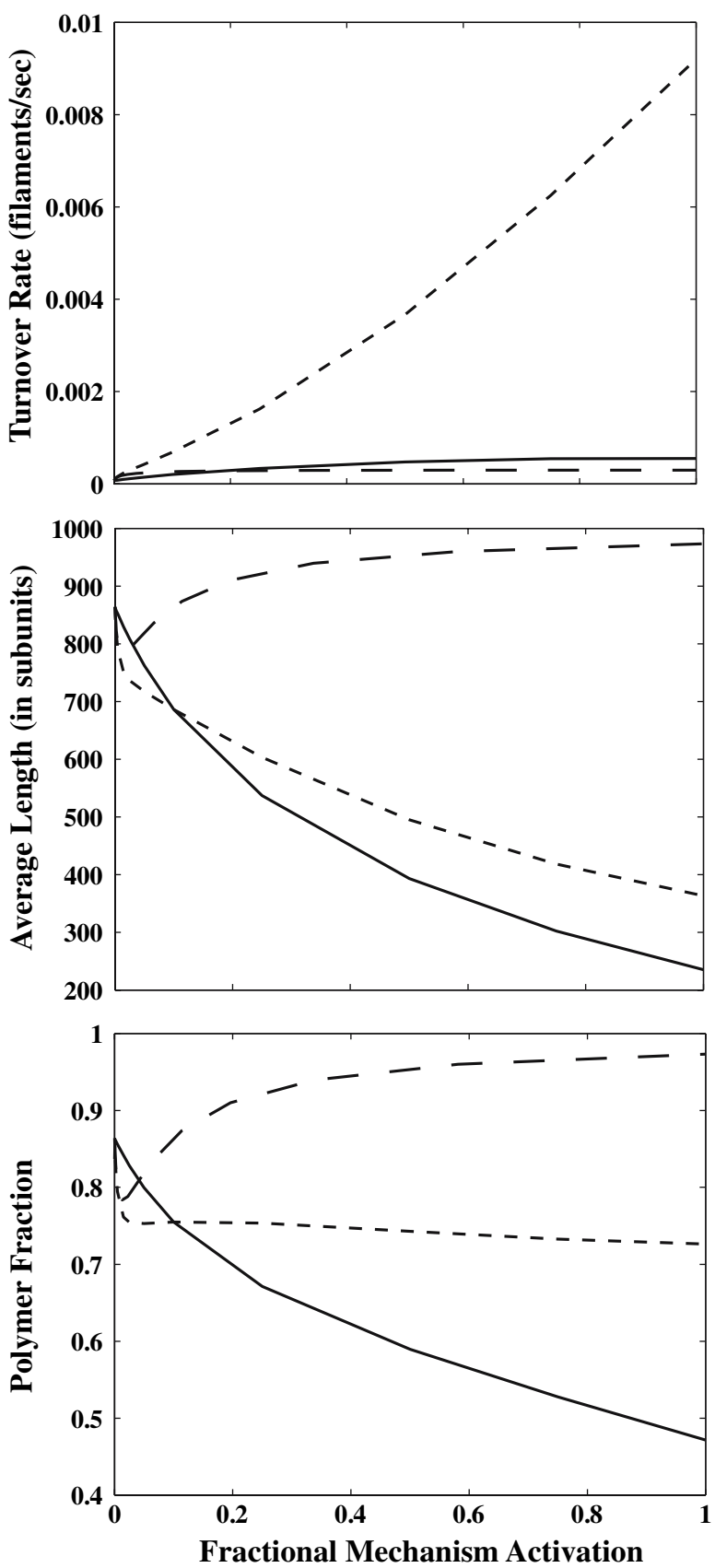

FIGURE 5. Synergy Between Cofilin and Proflin. Profilin concentrations are varied from 0 to $20 \mu \mathrm{M}$ and the long dash line represents profilin activity alone. Cofilin activity is represented by varying $k_{P D}^{-}$from 0.3 to $6 \mathrm{~s}^{-1}, k_{f D \cdot P i \rightarrow D}^{-}$from 0.0026 to $0.039 \mathrm{~s}^{-1}$, and $n$ from 0.1 to $0.2 \mu \mathrm{M}$ and the solid line represents cofilin activity alone. Varying cofilin and profilin together (short dash line) results in highly synergistic increases in filament turnover.

binding, and total actin content for bovine arterial endothelial cells (BAECs) in both a slowly crawling confluent state and a highly motile subconfluent state. While they did not determine profilin activity or quantify $k_{P D}^{-}$or $k_{f D \cdot P i \rightarrow D}^{-}$, McGrath et al. ${ }^{34}$ did find that cofilin was bound to filaments in both confluent 
and subconfluent cells, suggesting some activity in both states. Nonetheless, this wealth of information on a single cell type from a single set of experiments provides the best opportunity to compare our model predictions to data on real cells (Fig. 6). Using the measured values for $n$ and $\alpha$, selecting values for $k_{P D}^{-}$ and $k_{f D \cdot P i \rightarrow D}^{-}$consistent with cofilin activity, and assuming no profilin activity (also unmeasured), we find qualitative agreement between the measured shifts in turnover, length, and polymer fraction and model predictions. Furthermore, though the quantitative predictions depend on the particular parameter values used, we find excellent quantitative agreement for average length and polymer fraction for both cell activity levels, and turnover rate for confluent cells. The only significant disagreement is in the turnover rate for subconfluent cells, where the model prediction is about four-fold higher than the measured rate. Reducing the values of $k_{P D}^{-}$and $k_{f D \cdot P i \rightarrow D}^{-}$used for the subconfluent state lowers the turnover rate but increases the polymer fraction and average length; so there does not seem to be a single set of parameter values consistent with measurements which can quantitatively match all three state variables simultaneously for the subconfluent state.

\section{DISCUSSION}

In this report, we extended our recently published model of the actin cycle ${ }^{3}$ to allow a mechanistic interpretation of the higher order state variables estimable in cell experiments. We first demonstrate that flux, turnover, and hydrolysis are independent quantities despite some suggestions in literature that they are synonymous measures of cycle dynamics. We looked for signature effects to allow potential identification of underlying mechanism from higher order measurements of state variables, finding that some mechanisms have clear signatures and others do not. We examined the influence of thymosin $\beta 4$ and its interaction with profilin, as well as the synergistic effects of combining cofilin with profilin. Finally, we compared the state variable predictions of the extended actin cycle model with measurements from endothelial cells.

\section{Thymosin $\beta 4$, Profilin, and Cofilin}

Buffering of the monomeric actin pool by thymosin $\beta 4$ allows cells to maintain large pools of unpolymerized $\operatorname{actin}^{23}$ even in the presence of a low concentration of free barbed ends. We see this buffering in our model (compare Figs. 2 and 3) and find that it increases the effectiveness of accelerated
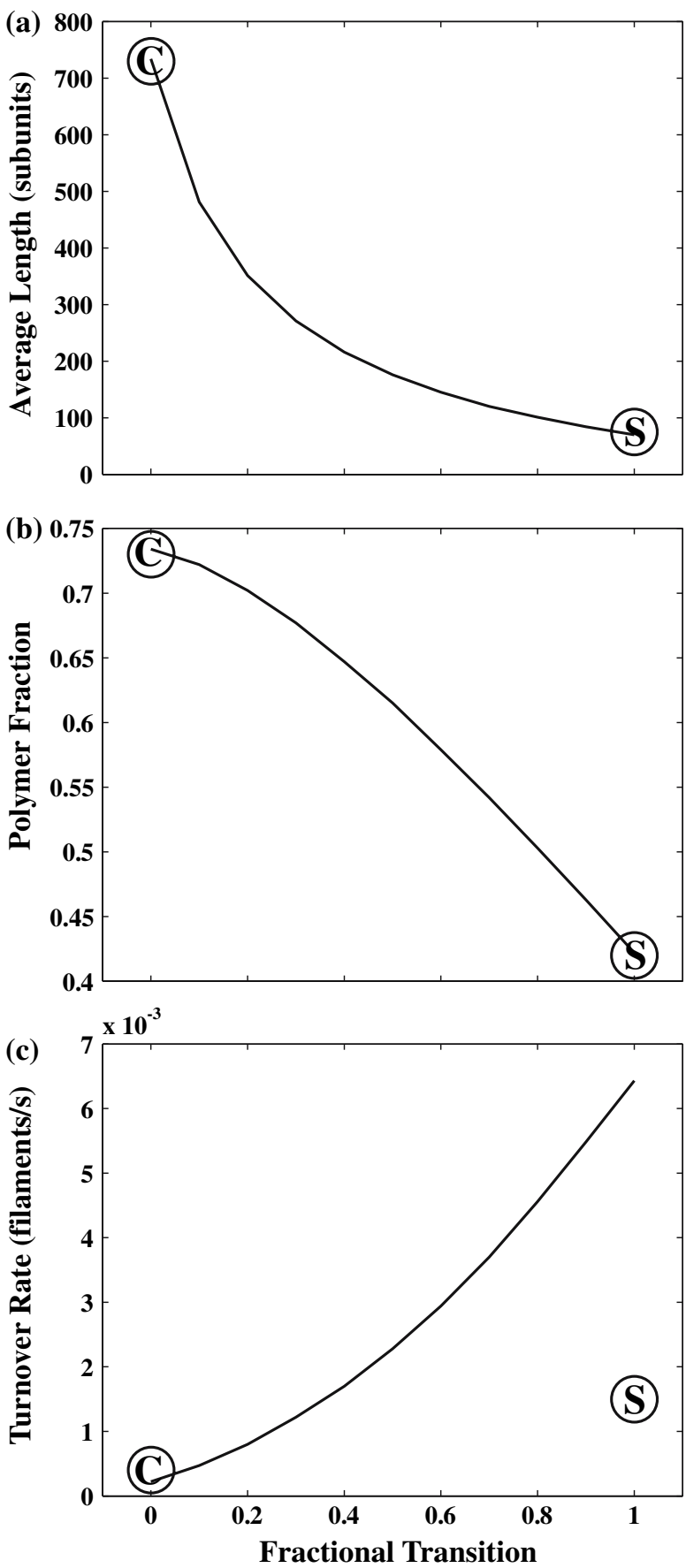

FIGURE 6. Model Comparison to Cellular Measurements. (a) Average length. (b) Polymer fraction. (c) Turnover rate. In each panel, the experimental measurement for confluent cells is marked by a circle with a $\mathrm{C}$ and the experimental measurement for subconfluent cells is marked by a circle with an $\mathrm{S}$. The line is the model prediction for the transition between the two states. Model variables transition as follows: $\alpha: 0.05$ to 0.3 , $n: 0.1 \mathrm{nM}$ to $0.6 \mathrm{nM}, k_{P D}^{-}: 1 \mathrm{~s}^{-1}$ to $6 \mathrm{~s}^{-1}, k_{f D \cdot P i \rightarrow D}^{-}: 0.0026 \mathrm{~s}^{-1}$ to $0.039 \mathrm{~s}^{-1}, P_{\text {tot }}$ : held at $0 \mu \mathrm{M}$.

depolymerization in increasing turnover rate and decreasing average length and polymer fraction. With all regulatory mechanisms active, the influence 
of thymosin $\beta 4$ is significantly reduced because the higher concentrations of barbed ends and profilin successfully compete for ATP-actin away and very little actin remains sequestered.

Goldschmidt-Clermont et al. ${ }^{18}$ first examined the interplay between thymosin $\beta 4$ and profilin, reporting that small amounts of profilin could overcome the inhibition of nucleotide exchange by thymosin $\beta 4$. The concentrations of actin, thymosin $\beta 4$, and profilin we use are much higher, but we obtain similar results. We find that only $0.3 \mu \mathrm{M}$ profilin is enough to convert the non-thymosin $\beta 4$-bound monomer pool from mostly ADP-bound to mostly ATP-bound. The model also predicts that thymosin $\beta 4$ is able to reverse the effects of small amounts of profilin on polymerization by sequestering ATP-actin monomers as quickly as they are produced by profilin's nucleotide exchange activity. While this effect has not been reported in the literature to date, our model provides guidelines for future experiments the might explore profilin-thymosin $\beta 4$ interplay in detail.

Another model prediction which has been confirmed experimentally is that cofilin and profilin are strongly synergistic in accelerating actin filament turnover. Didry et al. ${ }^{13}$ found a maximal $\sim 125$-fold increase in filament turnover with the combination of cofilin and profilin, and our model predicts a maximum 127-fold increase. The complementary activities of these two important actin cycle regulators provide drastically greater effects on filament turnover than either alone (Fig. 5), while the combination has more modest effects on polymer fraction and filament length.

\section{Signature Effects}

One of the results of this work is the table cataloguing the signature effects of five regulated mechanisms on average filament length, turnover rate, and polymer fraction (Table 2). How can this signature effects table be used? Consider the case of the endothelial cell data from McGrath et al., ${ }^{34}$ but instead of examining whether the model can produce the measured state variable shifts, we ask whether the signature effects table would allow us to guess the underlying mechanisms a prori. In endothelial cells transitioning from confluent to subconfluent states, the turnover rate increases about fourfold, the average length decreases about tenfold, and the polymer fraction decreases about two-fold. ${ }^{34}$ Referring to the table of signature effects, this pattern of changes ( $T R$ up, $L_{\text {avg }}$ down, and $P F$ down) suggests that underlying these changes are an increase in severing/nucleation (increasing filament number), an acceleration of depolymerization, and not much global profilin activity. In fact, McGrath et al. ${ }^{34}$ do find a large increase in filament number in this transition, as well as indications of cofilin activity in both states, which would contribute to severing, accelerated depolymerization, and acceleration of Pi release. In this case, at least, the signature effects do seem to allow some accurate inference of mechanism.

The ability to infer underlying mechanisms from state variable measurements is clearly limited. It is possible for combinations of mechanisms to mask one another (as noted for the combination of cofilin and profilin on average length and polymer fraction above), and not all underlying mechanisms have signature effects on state variables. Still, the signature effects table can be used as a general guideline to point an experimenter to the most likely cause of cytoskeleton state changes.

We find that two of the mechanisms considered, accelerated Pi release and barbed end uncapping, lack signature effects on the state variables. It is perhaps not significant that $k_{f D \cdot P i \rightarrow D}^{-}$does not have its own signature since the only known proteins which accelerate filament Pi release are those of the ADF/ cofilin family, which are also the only known accelerators of depolymerization. Further, accelerated Pi release is ineffective without accelerated ADP-actin depolymerization. ${ }^{3}$ Therefore, one can consider the signature of accelerated depolymerization to be a signature of cofilin activity and assume that accelerated $\mathrm{Pi}$ release accompanies accelerated depolymerization.

It is more significant that the degree of barbed end capping does not seem to have a strong effect on state variables. Experimentalists often describe uncapping barbed ends in cells as leading to "explosive" growth upon cellular activation. ${ }^{45}$ While our steady state model cannot comment on transient effects, uncapping alone does not seem to drastically change the average length of filaments or fraction of actin polymerized at steady state. The activated steady state we see may correspond to the persistent crawling phase of neutrophils and the soil amoeba Dictyostelium discoideum following chemoattractant gradients, for example. Both these cells experience an early burst of polymerization upon first contact with a chemoattractant, then polarize and crawl steadily up the gradient. ${ }^{42}$ During the steady crawling phase, the fraction of polymerized actin returns to near pre-stimulation levels. ${ }^{11,22}$ It is sometimes assumed that this return is due to eventual recapping of filaments to their prestimulation levels, but our model results suggest that recapping is not necessary as the steady state polymer fraction is not a strong indicator of the fraction of free barbed ends. 


\section{Model Agreements and Shortcomings}

The relevance of a spatially homogeneous, steady state model to the complex actin dynamics in living cells is debatable. Most cells do exhibit periods of persistent motion that may reasonably be described as steady state, but it is clear that spatial and temporal inhomogeneities are also essential. Despite this, our model seems to be of some use in connecting regulated mechanisms to cytoskeletal state variables, even for living endothelial cells. McGrath et al. ${ }^{34}$ measured all three state variables and some of the underlying mechanism values in endothelial cells as they transitioned from a slowly crawling confluent state to a highly motile subconfluent state. It is important to note that all measurements by McGrath et al. should be considered cellular averages because the measurement techniques had at best, a low spatial resolution. Using the measured changes in underlying mechanisms and reasonable guesses for unmeasured values, we asked whether the model could reproduce the measured transitions in state variables (Fig. 6). Generally, we found agreement between model predictions and measured values; the only quantitative discrepancy was the model's prediction for turnover rate in subconfluent cells, which was about four-fold higher than cellular measurements.

Activation for neutrophils, Dictyostelium, and platelets leads to a massive increase in the polymer fraction, so it may seem surprising that activation of endothelial cells leads to a significant decrease in the polymer fraction. However, it should be noted that this rapid increase is reversed within seconds (for Dictyostelium) or minutes (for neutrophils) and the polymer fraction is only permanently increased for non-motile platelets. In addition, a decrease in polymer fraction between confluent and crawling endothelial cells (0.62--0.48) has been observed previously by Gabbiani et al. ${ }^{16}$

Profilin is an essential protein in every eukaryotic cell type where it has been tested, so it seems odd that we get our best agreement with experimental data by omitting all profilin activity. Neither McGrath et al. ${ }^{34}$ nor Gabbiani et al. ${ }^{16}$ measured profilin concentrations, but it was certainly present in these cells. The solution to this conundrum may lie in the cytoplasmic localization of profilin, something which is beyond the capability of the current model to represent. For example, profilin has been shown to associate with the membrane phosphoinositides PIP and PIP $_{2}$ and this binding both localizes profilin to the plasma membrane ${ }^{40}$ and inhibits actin binding. ${ }^{30}$ So, it is certainly possible there actually was very little free profilin available in the cytoplasm of the endothelial cells to influence the bulk actin cycle.

More generally, for many cells it has been shown that different parts of the actin cycle are spatially segregated. For example, in highly motile keratocytes, filament generation and assembly take place primarily in a narrow band near the leading edge of the cell, while depolymerization of the network occurs everywhere and cofilin is excluded from the leading edge. ${ }^{45}$ It is again impossible to investigate the effects or causes of this segregation with our current model. The good agreement of our model with endothelial cell data suggests that it has some utility as is, but even greater utility will come with an advanced model that respects the geometry and dynamics of real cells. Existing spatially resolved models of actin dynamics ${ }^{26,36}$ have contributed to the understanding of actin dynamics near the leading edge of cells, but, except for capping, they lack the consideration of actin cycle regulation which is the real strength of our model. The next steps in the development of our model are to solve the model equations in time so that transient effects can be examined and to incorporate a simplified cellular geometry so that spatial effects can be investigated. Ultimately, we envision a future model which also encompasses the mechanical functions of the actin cytoskeleton. Local network viscoelasticities are available from intracellular microrheology measurements in cells ${ }^{59}$ and reconstituted actin gels. ${ }^{33}$ These measurements would provide additional state variables to help identify actin remodeling mechanisms in live cell measurements. To be clear, our goal is not a model which is accurate in all molecular details, but the simplest model that correctly predicts secondary parameters from primary modifications of actin filaments. Correlating secondary parameters with cell behavior would then provide a continuous line of evidence connecting macroscopic behavior and regulated activities on filaments. In combination with drugs that influence these control points, the model then becomes an essential part of a strategy for controlling cell behavior in complex environments.

\section{APPENDIX A}

The following tables repeat the equations, variable definitions and values used in Bindschadler et al. ${ }^{3}$ 
TABLE A1. Parameters with default values.

\begin{tabular}{|c|c|c|c|}
\hline Constant & Description & Value & References \\
\hline$k_{b T}^{+}$ & ATP-G-actin association at barbed ends & $11.6 /(\mu \mathrm{M} \mathrm{s})$ & Pollard ${ }^{43}$ \\
\hline$k_{b D \cdot P i}^{+}$ & ADP.Pi-G-actin associationat barbed ends & $11.6 /(\mu \mathrm{M} \mathrm{s})$ & Equivalence to ATPactin \\
\hline$k_{b D}^{+}$ & ADP-G-actin association at barbed ends & $3.8 /(\mu \mathrm{M} \mathrm{s})$ & Pollard ${ }^{43}$ \\
\hline$k_{p T}^{+}$ & ATP-G-actin association at pointed ends & $1.3 /(\mu \mathrm{M} \mathrm{s})$ & Pollard ${ }^{43}$ \\
\hline$k_{p D \cdot P i}^{+}$ & ADP.Pi-G-actin association at pointed ends & $1.3 /(\mu \mathrm{M} \mathrm{s})$ & Equivalence to ATPactin \\
\hline$k_{p D}^{+}$ & ADP-G-actin association at pointed ends & $0.16(\mu \mathrm{M} s)$ & Pollard ${ }^{43}$ \\
\hline$k_{b T}^{-}$ & ATP-G-actin disassociation at barbed ends & $1.4 / \mathrm{s}$ & Pollard ${ }^{43}$ \\
\hline$k_{b D \cdot P i}^{-}$ & ADP.Pi subunit disassociation at barbed ends & $1.4 / \mathrm{s}$ & Equivalence to ATPactin \\
\hline$k_{b D}^{-}$ & ADP subunit disassociationat barbed ends & $7.2 / \mathrm{s}$ & Pollard ${ }^{46}$ \\
\hline$k_{p T}^{-}$ & ATP subunit disassociation at pointed ends & $0.8 / \mathrm{s}$ & Pollard ${ }^{43}$ \\
\hline$k_{p D \cdot P i}^{-}$ & ADP.Pi subunit disassociation at pointed ends & $0.8 / \mathrm{s}$ & Equivalence to ATPactin \\
\hline$k_{p D}^{-}$ & ADP subunit disassociation at pointed ends & $0.3 / \mathrm{s}$ & Calculated from Pollard ${ }^{43}$ \\
\hline$k_{P D}^{-}$ & Profilin-ADP-G-actin complex dissociation rate & $0.65 / \mathrm{s}$ & $\begin{array}{l}\text { Based on equilibrium constant } \\
\text { from Selden et al. }{ }^{49}\end{array}$ \\
\hline$k_{P D}^{+}$ & Profiling-ADP-G-actin complex association rate & $1 /(\mu \mathrm{M} \mathrm{s})$ & Assigned \\
\hline$k_{P}^{-}$ & Profilin-ATP-G-actin complex dissociation rate & $0.6 / \mathrm{s}$ & $\begin{array}{l}\text { Based on equilibrium constant } \\
\text { from Selden et al. }{ }^{49}\end{array}$ \\
\hline$k_{P D \cdot P i}^{-}$ & Profilin-ADP.Pi-G-actin complex dissociation rate & $0.6 / \mathrm{s}$ & Equivalence to ATPactin \\
\hline$k_{P T}^{+}$ & Profilin ATP-G-actin association rate & $1 /(\mu \mathrm{M} \mathrm{s})$ & Assigned \\
\hline$k_{P D \cdot P i}^{+}$ & Profilin, ADP.Pi-G-actin associationrate & $1 /(\mu \mathrm{M} \mathrm{s})$ & Equivalence to ATPactin \\
\hline$k_{m D}>T$ & Rate of ADP exchange for ATP on free monomer & $0.01 / \mathrm{s}$ & Selden et al. ${ }^{49}$ \\
\hline$k_{P D}>T$ & Rate of ADP exchange for ATP on profilin-actin & $1.40 / \mathrm{s}$ & Selden et al. ${ }^{49}$ \\
\hline$k_{P D \cdot P i}>T$ & Rate of ADP.Pi exchange for ATP on profilin-actin & $0.08 / \mathrm{s}$ & Equivalence to ATPactin \\
\hline$K_{T>D \cdot P i}$ & Rate of ATP hydrolysis on filaments & $0.3 / \mathrm{s}$ & Blanchoin and Pollard ${ }^{4}$ \\
\hline$k_{f D \cdot P i}>D$ & Rate of $\mathrm{Pi}$ release from ADP.Pi subunits & $0.0026 / \mathrm{s}$ & Melki et al. ${ }^{35}$ \\
\hline$k_{m D \cdot P i}>D$ & Rate of Pi release from ADP.Pi-G-actin & $10,000 / \mathrm{s}$ & Immediate release of $\mathrm{Pi}$ on $\mathrm{G}$-actin \\
\hline$K_{B D}$ & $\begin{array}{l}\text { Equilibrium disassociation constant for } \\
\text { thymosin B4 and ADP-G-actin }\end{array}$ & $100 \mu \mathrm{M}$ & $\begin{array}{l}k+B D=1 /(\mu \mathrm{M} \mathrm{s}) \text { and } k-B D=100 / \mathrm{s} \\
\text { assigned; } k_{B D} \text { from Carlier et al. }{ }^{10}\end{array}$ \\
\hline$K_{B T}$ & $\begin{array}{l}\text { Equilibrium disassociation constant for } \\
\text { thymosin B4 and ATP-G-actin }\end{array}$ & $0.9 \mu \mathrm{M}$ & $\begin{array}{l}k+B T=1 /(\mu \mathrm{M} \mathrm{s}) \text { and } k-B T=/ 0.9 \mathrm{~s} \\
\text { assigned; } K_{B T} \text { from Kang et al. }{ }^{25}\end{array}$ \\
\hline$K_{B D \cdot P i}$ & $\begin{array}{l}\text { Equilibrium disassociation constant } \\
\text { for thymosin B4 and ADP.Pi-G-actin }\end{array}$ & $0.9 \mu \mathrm{M}$ & Equivalence to ATPactin \\
\hline
\end{tabular}

TABLE A2. Equations of the actin cycle.

E1 no net assembly of filaments

$0=\alpha[n]\left(k_{\mathrm{b} T}^{+}([P a f]+[a f])-k_{\mathrm{b} T}^{-} g_{\mathrm{bT}}(1)+k_{\mathrm{bD} \cdot \mathrm{Pi}_{\mathrm{i}}}([a d p i]+[P a d p \mathrm{i}])-k_{\mathrm{bD} \cdot \mathrm{Pi}}^{-} g_{\mathrm{bD} \cdot \mathrm{Pi}(1)}+k_{\mathrm{bD}}^{+}([a d]+[P a d])-k_{\mathrm{bD}}^{-} g_{\mathrm{bD}(1)}\right)$

$+\beta[n]\left(k_{p T}^{+}[a f]-k_{p T}^{-} g_{p T(1)}+k_{\mathrm{pD} \cdot \mathrm{Pi}}^{+}[a d p i]-k_{\mathrm{pD} \cdot \mathrm{Pi}}^{-} g_{\mathrm{pD} \cdot \mathrm{Pi}(1)}+k_{\mathrm{pD}}^{+}[a d]-k_{\mathrm{pD}}^{-} g_{\mathrm{pD}(1)}\right)$

E2 no net formation of ADP-G-Actin

$0=\beta[n]\left(k_{\mathrm{pD}}^{-} g_{\mathrm{pD}(1)}-k_{\mathrm{pD}}^{+}[\mathrm{ad}]\right)+\alpha[n]\left(k_{\mathrm{bD}}^{-} g_{\mathrm{bD}(1)}-k_{\mathrm{bD}}^{+}([\mathrm{ad}]+[P a d])\right)-k_{m D}>T[\mathrm{ad}]+k_{\mathrm{pD}}^{-}[P a d]-k_{\mathrm{pD}}^{+}[P][\mathrm{ad}]+k_{\mathrm{BD}}^{-}[\mathrm{Bad}]-k_{\mathrm{BD}}^{+}[B][a d]$

$+k_{m D \cdot P i}>D[a d p i]$

E3 no net formation of ADP $\cdot$ Pi-G-actin

$0=\beta[n]\left(k_{\mathrm{pD} \cdot \mathrm{Pi}}^{-} g_{\mathrm{pD} \cdot \mathrm{Pi}(1)}-k_{\mathrm{pD} \cdot \mathrm{Pi}}^{+}[a d p i]\right)+\alpha[n]\left(k_{\mathrm{bD} \cdot \mathrm{Pi}}^{-} g_{\mathrm{bD} \cdot \mathrm{Pi}(1)}-k_{\mathrm{bD} \cdot \mathrm{Pi}}^{+}([a d p i]+[\right.$ Padpi $\left.])\right)-k_{\mathrm{PD} \cdot \mathrm{Pi}}^{-}[$Padpi $]-k_{\mathrm{PD} \cdot \mathrm{Pi}}^{+}[\mathrm{P}][a d p \mathrm{i}]$

$+k_{\mathrm{BD} \cdot \mathrm{Pi}}^{-}[$Badpi $]-k_{\mathrm{BD} \cdot \mathrm{Pi}}^{+}[\mathrm{B}][\mathrm{adpi}]-k_{m D \cdot \mathrm{Pi}>D}[\mathrm{adpi}]$

E4 conservation of actin

Atot $=[\mathrm{at}]+[\mathrm{ad}]+[\mathrm{adp}]+[\mathrm{f}]+[\mathrm{Pat}]+[\mathrm{Pad}]+[$ Padpi $]+[\mathrm{Bat}]+[\mathrm{Bad}]+[$ Badpi $]$

E5 (barbed: $\boldsymbol{e}=\boldsymbol{b} ; \gamma=\alpha ; \varepsilon=1$ ) E6 (pointed: $\boldsymbol{e}=\boldsymbol{p} ; \gamma=\beta ; \varepsilon=0$ ) no net formation of ATP-bound termini

$0=\gamma\left(k_{\mathrm{eT}}^{+}([a f]+\varepsilon[P a f])\left(1-g_{\mathrm{eT}(1)}\right)-k_{\mathrm{eT}}^{-} g_{\mathrm{eT}(1)}\right)\left(1-g_{\mathrm{eT}(2)}\right)+\left(k_{\mathrm{eD}}^{-} g_{\mathrm{eD}(1)}+k_{\mathrm{eD} \cdot \mathrm{Pi}}^{-} g_{\mathrm{eD} \cdot \mathrm{Pi}(1)}\right) g_{\mathrm{eT}(2)}-\left(k_{\mathrm{eD}}^{+}([a d]+\varepsilon[P a d])\right.$

$+k_{\mathrm{eD} \cdot \mathrm{Pi}}^{+}([\mathrm{adpi}]+\varepsilon[$ Padpi $\left.\left.])\right) g_{\mathrm{eT}(1)}\right) k_{T>D \cdot P \mathrm{i}} g_{\mathrm{eT}(1)}$

E7 (barbed: $e=b ; \gamma=\alpha ; \varepsilon=1$ ) E8 (pointed: $e=p ; \gamma=\beta ; \varepsilon=0$ ) no net formation of ADP-bound termini

$0=\gamma\left(k_{\mathrm{eD}}^{+}([a d]+\varepsilon[P a d])\left(1-g_{\mathrm{eD}(1)}\right)-k_{\mathrm{eD}}^{-} g_{\mathrm{eD}(1)}\left(1-g_{\mathrm{eD}(2)}\right)\left(k_{\mathrm{eT}}^{+}([a f]+\varepsilon[P a f])+k_{\mathrm{eD} \cdot \mathrm{Pi}}^{+} g_{\mathrm{eD}(1)}([a d p i]+\varepsilon[P a d p i])\right) g_{\mathrm{eD}(1)}+\left(k_{\mathrm{eT}}^{-} g_{\mathrm{eT}(1)}\right.\right.$

$\left.\left.+k_{\mathrm{eD} \cdot \mathrm{Pi}}^{-} g_{\mathrm{eD} \cdot \mathrm{pi}(1)}\right) g_{\mathrm{eD}(2)}\right)+k_{f D \cdot \mathrm{Pi}}>D g_{\mathrm{eD} \cdot \mathrm{Pi}(1)}$

E9 (barbed: $e=b$ ) E10 (pointed: $e=p$ ) terminal subunits are bound to 1 of 3 Nucleotides

$1=g_{\mathrm{eD} \cdot \mathrm{pi}(1)}+g_{\mathrm{eD}(1)}+g_{\mathrm{eT}(1)}$

E11 (barbed: $\boldsymbol{e}=\boldsymbol{b} ; \gamma=\alpha ; \varepsilon=1) \mathrm{E} 12$ (pointed: $\boldsymbol{e}=\boldsymbol{p} ; \gamma=\beta ; \varepsilon=0$ ) no net production of ATP at ith subunit $\left(1<\mathrm{i}<\mathrm{i}^{\star}\right)$

$0=\gamma\left(\left(k_{\mathrm{eT}}^{-} g_{\mathrm{eT}(1)}+k_{\mathrm{eD} \cdot \mathrm{Pi}}^{-} g_{\mathrm{eD} \cdot \mathrm{Pi}(1)}+k_{\mathrm{eD}}^{-} g_{\mathrm{eD}(1)}\right)\left(g_{\mathrm{eT}(\mathrm{i}+1)}-g_{\mathrm{eT}(\mathrm{i})}\right)+\left(k_{\mathrm{eT}}^{+}([a f]+\varepsilon[\right.\right.$ Paf $])+k_{\mathrm{eD} \cdot \mathrm{Pi}_{\mathrm{i}}}([a d p i]+\varepsilon[$ Padpi $])+k_{\mathrm{eD}}^{+}([a d]$

$\left.+\varepsilon[P a d]))\left(g_{\mathrm{eT}(\mathrm{i}-1)}-g_{\mathrm{eT}(\mathrm{i})}\right)\right)-k_{T}>D \cdot \mathrm{Pi} g_{\mathrm{eT}(\mathrm{i})}$ 
TABLE A2. Continued

E13 (barbed: $\boldsymbol{e}=\boldsymbol{b} ; \gamma=\alpha ; \varepsilon=1$ ) E14 (pointed: $\boldsymbol{e}=\boldsymbol{p} ; \gamma=\beta ; \varepsilon=0$ ) no net production of ADP at ith subunit $\left(1<\mathrm{i}<\mathrm{i}^{*}\right)$

$0=\gamma\left(\left(k_{\mathrm{eT}}^{-} g_{\mathrm{eT}(1)}+k_{\mathrm{eD} \cdot \mathrm{Pi}}^{-} g_{\mathrm{eD} \cdot \mathrm{Pi}(1)}+k_{\mathrm{eD}}^{-} g_{\mathrm{eD}(1)}\right)\left(g_{\mathrm{eD}(i+1)}-g_{\mathrm{eD}(\mathrm{i})}\right)+\left(k_{\mathrm{eT}}^{+}([a t]+\varepsilon[\right.\right.$ Paf $])+k_{\mathrm{eD} \cdot \mathrm{Pi}}^{+}([a d p i]+\varepsilon[$ Padpi $])+k_{\mathrm{eD}}^{+}([a d]$

$\left.+\varepsilon[P a d]))\left(g_{\mathrm{eD}(\mathrm{i}-1)} g_{\mathrm{eD}(\mathrm{i})}\right)\right)-k_{f \mathrm{D} \cdot \mathrm{Pi}}>D g_{\mathrm{eD} \cdot \mathrm{Pi}(1)}$

E15 (barbed: $e=p$ ) E16 (pointed: $e=p$ ) ith subunits are bound to 1 of 3 nucleotides

$1=g_{\mathrm{eT}(\mathrm{i})}+g_{\mathrm{eD} \cdot \mathrm{Pi}(\mathrm{i})}+g_{\mathrm{eD}(\mathrm{i})}$

E17 E18 continutity at $i^{*}$

$g_{\mathrm{bD}(\mathrm{i})}=g_{\mathrm{pD}(\mathrm{i})} g_{\mathrm{bD} \cdot \mathrm{Pi}(\mathrm{i})}=g_{\mathrm{pD} \cdot \mathrm{Pi}(\mathrm{i})}$

E19 (barbed: $e=p$ ) E20 (pointed: $e=p$ ) ith subunits are bound to 1 of 3 nucleotides

$1=g_{\mathrm{eT}(\mathrm{i})}+g_{\mathrm{eD} \cdot \mathrm{P}(\mathrm{i})}+g_{\mathrm{eD}(\mathrm{i})}$

E21 E22 continuity at $i$ *

$g_{\mathrm{eD}(\mathrm{i})}$ and $g_{\mathrm{eD} \cdot \mathrm{Pi}(\mathrm{i})}$ lie on a cubic flanking $\mathrm{i}^{*}$

E23 no net formation of profilin/ATP-actin

$0=k_{p T}^{+}[a f][P]-k_{\mathrm{PT}}^{-}[P a t]+k_{\mathrm{PD}}>T[P a d]-\alpha k_{\mathrm{bT}}^{+}[P a f][n]+k_{\mathrm{PD} \cdot \mathrm{Pi}}>T[P a d p \mathrm{i}]$

E24 no net formation of profilin/ADP $\cdot \mathrm{Pi}$ actin

$0=k_{\mathrm{DD} \cdot \mathrm{Pi}}^{+}[$adpi $][P]-k_{\mathrm{PD} \cdot \mathrm{Pi}}^{-}[$Padpi $]-\alpha k_{\mathrm{bD} \cdot \mathrm{Pi}}^{+}[$Padpi $][n]-k_{\mathrm{PD} \cdot \mathrm{Pi}}>T[$ Padpi $]$

E25 no net formation of free profilin

$0=\alpha[n]\left(k_{\mathrm{bT}}^{+}[P a f]+k_{\mathrm{bD} \cdot \mathrm{Pi}}^{+}[P a d p i]+k_{\mathrm{bD}}^{+}[P a d]\right)-k_{\mathrm{PT}}^{+}[\mathrm{af}][P]-k_{\mathrm{PD} \cdot \mathrm{Pi}}^{+}[\mathrm{adpi}][P]-k_{\mathrm{DD}}^{+}[\mathrm{ad}][P]+k_{\mathrm{PT}}^{-}[P a f]+k_{\mathrm{PD} \cdot \mathrm{Pi}^{-}}^{-}[P a d p i]+k_{\mathrm{PD}}^{-}[P a d]$

E26 conservation of profilin

$P_{\text {tot }}=[P]+[P a t]+[P a d]+[$ Padpi $]$

E27 thymosin $\beta 4$ ATP-G-actin equilibrium

$K_{\mathrm{BT}}=([a t][B]) /[\mathrm{Bat}]$

E28 thymosin $\beta 4$ ADP-G-actin equilibrium

$K_{\mathrm{BD}}=([\mathrm{ad}][\mathrm{B}]) /[\mathrm{Bad}]$

E29 thymosin $\beta 4$ ADP · pi-G-actin equilibrium

$K_{\mathrm{BD} \cdot \mathrm{Pi}}=([$ adpi $][B]) /[$ Badpi $]$

E30 conservation of thymosin $\beta 4$

$B_{\text {tot }}=[B]+[B a t]+[$ Badpi $]+[B a d]$

TABLE A3. Variable and parameter definitions.

\begin{tabular}{ll}
\hline Variable & Description \\
\hline$a t$ & Concentration of ATP-bound unpolymerized actin \\
ad & Concentration of ADP-bound unpolymerized actin \\
adpi & Concentration of ADP.Pi-bound unpolymerized actin \\
$f$ & Concentration of actin in filaments \\
$n$ & Concentration of filaments \\
$A_{\text {tot }}$ & Total concentration of actin \\
$B_{\text {tot }}$ & Total concentration of thymosin $\beta$ s \\
$P_{\text {tot }}$ & Total concentration of profilin \\
$B$ & Concentration of free thymosin $\beta$ s \\
$B a t$ & Concentration of ATP-bound monomer Complexed with thymosin $\beta$ s \\
$B a d$ & Concentration of ADP-bound monomer complexed with thymosin $\beta$ s \\
$B a d p i$ & Concentration of ADP.Pi-bound monomer complexed with thymosin $\beta$ s \\
$P$ & Concentration of free profilin \\
$P a t$ & Concentration of free ATP-bound monomer complexed with profilin \\
$P a d$ & Concentration of free ADP-bound monomer complexed with profilin \\
$P a d p i$ & Concentration of free ADP.Pi-bound monomer complexed with profilin \\
$\alpha$ & Fraction of barbed ends capped \\
$\beta$ & Fraction of pointed ends capped \\
$g b T(i)$ & Fraction of subunits at position $i$ near filament barbed ends that are bound to ATP \\
$g b D \cdot P(i)$ & Fraction of subunits at position $i$ near filament barbed ends that are bound to ADP.Pi \\
$g b D(i)$ & Fraction of subunits at position $i$ near filament barbed ends that are bound to ADP \\
$g P T(i)$ & Fraction of subunits at position $i$ near filament pointed ends that are bound to ATP \\
$g p D \cdot P i(i)$ & Fraction of subunits at position $i$ near filament pointed ends that are bound to ADP.Pi \\
$g p D(i)$ & Fraction of subunits at position $i$ near filament pointed ends that are bound to ADP \\
$q$ & Rate of subunit flux \\
\hline &
\end{tabular}




\section{REFERENCES}

${ }^{1}$ Bamburg, J. R. and D. Bray. Distribution and cellular localization of actin depolymerizing factor. J. Cell Biol. 105:2817-2825, 1987.

${ }^{2}$ Barkalow, K., W. Witke, D. J. Kwiatkowski, and J. H. Hartwig. Coordinated regulation of platelet actin filament barbed ends by gelsolin and capping protein. J. Cell Biol. 134:389-399, 1996.

${ }^{3}$ Bindschadler, M., E. A. Osborn, C. F. Dewey Jr., and J. L. McGrath. A mechanistic model of the actin cycle. Biophys. J. 86:2720-2739, 2004.

${ }^{4}$ Blanchoin, L. and T. D. Pollard. Hydrolysis of atp by polymerized actin depends on the bound divalent cation but not profilin. Biochemistry 41:597-602, 2002.

${ }^{5}$ Blanchoin, L. and T. D. Pollard. Mechanism of interaction of acanthamoeba actophorin (adf/cofilin) with actin filaments. J. Biol. Chem. 274:15538-15546, 1999.

${ }^{6}$ Bray, D. and C Thomas. The actin content of fibroblasts. Biochem. J. 147:221-228, 1975.

${ }^{7}$ Brenner, S. L. and E. D. Korn. The effects of cytochalasins on actin polymerization and actin atpase provide insights into the mechanism of polymerization. J. Biol. Chem. 255:841-844, 1980.

${ }^{8}$ Cano, M., D. Lauffenburger, and S. Zigmond. Kinetic analysis of $\mathrm{f}$-actin depolymerization in polymorphonuclear leukocyte lysates indicates that chemoattractant stimulation increases actin filament number without altering the filament length distribution. J. Cell Biol. 115:677-687, 1991.

${ }^{9}$ Carlier, M., V. Laurent, J. Santolini, R. Melki, D. Didry, G.-X. Xia, Y. Hong, N.-H. Chua, and D. Pantaloni. Actin depolymerizing factor (adf/cofilin) enhances the rate of filament turnover: Implication in actin based motility. $J$. Cell Biol. 136:1307-1322, 1997.

${ }^{10}$ Carlier, M.-F., C. Jean, K. Rieger, M. Lenfant, and D. Pantaloni. Modulation of the interaction between g-actin and thymosin b4 by the atp/adp ratio: Possible implication in the regulation of actin dynamics. Proc. Natl. Acad. Sci. U.S.A. 90:5034-5038, 1993.

${ }^{11}$ Condeelis, J. and A. L. Hall. Measurement of actin polymerization and cross-linking in agonist-stimulated cells. Methods Enzymol. 196:486-496, 1991.

${ }^{12}$ Devineni, N., L. S. Minamide, M. Niu, D. Safer, R. Verma, J. R. Bamburg, and V. T. Nachmias. A quantitative analysis of g-actin binding proteins and the g-actin pool in developing chick brain. Brain Res. 823:129-140, 1999.

${ }^{13}$ Didry, D., M. F. Carlier, and D. Pantaloni. Synergy between actin depolymerizing factor/cofilin and profilin in increasing actin filament turnover. J. Biol. Chem. 273:25602-25611, 1998.

${ }^{14}$ dos Remedios, C. G., D. Chhabra, M. Kekic, I. V. Dedova, M. Tsubakihara, D. A. Berry, and N. J. Nosworthy. Actin binding proteins: Regulation of cytoskeletal microfilaments. Physiol Rev 83:433-473, 2003.

${ }^{15}$ Falet, H., K. M. Hoffmeister, R. Neujahr, J. E. Italiano Jr., T. P. Stossel, F. S. Southwick, and J. H. Hartwig. Importance of free actin filament barbed ends for $\operatorname{arp} 2 / 3$ complex function in platelets and fibroblasts. Proc. Natl. Acad. Sci. USA 99:16782-16787, 2002.

${ }^{16}$ Gabbiani, G., F. Gabbiani, R. L. Heimark, and S. M. Schwartz. Organization of actin cytoskeleton during early endothelial regeneration in vitro. J. Cell. Sci. 66:39-50, 1984.

${ }^{17}$ Goldschmidt-Clermont, P., L. Machesky, S. Doberstein, and T. Pollard. Mechanism of the interaction of human platelet profilin with actin. J. Cell Biol. 113:1081-1089, 1991.

${ }^{18}$ Goldschmidt-Clermont, P. J., M. I. Furman, D. Wachsstock, D. Safer, V. T. Nachmias, and T. D. Pollard. The control of actin nucleotide exchange by thymosin beta 4 and profilin. A potential regulatory mechanism for actin polymerization in cells. Mol. Biol. Cell 3:1015-1024, 1992.

${ }^{19}$ Gutsche-Perelroizen, I., J. Lepault, A. Ott, and M. Carlier. Filament assembly from profilin-actin. J. Biol. Chem. 274:6234-6243, 1999.

${ }^{20}$ Hannappel, E. and M. van Kampen. Determination of thymosin beta 4 in human blood cells and serum. $J$. Chromatogr. 397:279-285, 1987.

${ }^{21}$ Hartwig, J. H., G. M. Bokoch, C. L. Carpenter, P. A. Janmey, L. A. Taylor, A. Toker, and T. P. Stossel. Thrombin receptor ligation and activated rac uncap actin filament barbed ends through phosphoinositide synthesis in permeabilized human platelets. Cell 82:643-653, 1995.

${ }^{22}$ Howard, T. H. and C. O. Oresajo. The kinetics of chemotactic peptide-induced change in f-actin content, f-actin distribution, and the shape of neutrophils. J. Cell Biol. 101:1078-1085, 1985.

${ }^{23}$ Huff, T., C. S. Muller, A. M. Otto, R. Netzker, and E. Hannappel. Beta-thymosins, small acidic peptides with multiple functions. Int. J. Biochem. Cell Biol. 33:205-220, 2001.

${ }^{24}$ Isenberg, G., U. Aebi, and T. D. Pollard. An actin-binding protein from acanthamoeba regulates actin filament polymerization and interactions. Nature 288:455-459, 1980.

${ }^{25}$ Kang, F., D. Purich, and F. Southwick. Profilin promotes barbed-end actin filaments assembly without lowering the critical concentration. J. Biol. Chem. 274:36963-36972, 1999.

${ }^{26}$ Karakozova, M., M. Kozak, C. C. Wong, A. O. Bailey, J. R. Yates 3rd, A. Mogilner, H. Zebroski, and A. Kashina. Arginylation of beta-actin regulates actin cytoskeleton and cell motility. Science 313:192-196, 2006.

${ }^{27}$ Kouyama, T. and K. Mihashi. Fluorimetry study of $n$-(1pyrenyl)iodoacetamide-labelled f-actin. Local structural change of actin protomer both on polymerization and on binding of heavy meromyosin. Eur. J. Biochem. 114:33-38, 1981.

${ }^{28}$ Kovar, D. R., E. S. Harris, R. Mahaffy, H. N. Higgs, and T. D. Pollard. Control of the assembly of atp- and adpactin by formins and profilin. Cell 124:423-435, 2006.

${ }^{29}$ Kreis, T., B. Geiger, and J. Schlessinger. Mobility of microinjected rhodamine actin within living chicken gizzard cells determined by fluorescence photobleaching recovery. Cell 29:835-845, 1982.

${ }^{30}$ Lassing, I. and U. Lindberg. Specific interaction between phosphatidyl-inositol 4,5-bisphosphate and profilactin. Nature 314:472-474, 1985.

${ }^{31}$ Maciver, S., H. Zot, and T. Pollard. Characterization of actin filament severng by actophorin from acanthamoeba castellanii. J. Cell. Biol. 115:1621-1620, 1991.

${ }^{32}$ McGough, A., B. Pope, W. Chiu, and A. Weeds. Cofilin changes the twist of f-actin: Implications for actin filament dynamics and cellular function. J Cell Biol 138:771-781, 1997.

${ }^{33}$ McGrath, J., J. Hartwig, and S. Kuo. The mechanics of factin microenvironements depend on the chemistry of probing surfaces. Biophys. J. 79:3258-3266, 2000.

${ }^{34}$ McGrath, J., E. Osborn, Y. Tardy, C. Dewey Jr., and J. Hartwig. Regulation of the actin cycle in vivo by actin filament severing. PNAS 97:6532-6537, 2000. 
${ }^{35}$ Melki, R., S. Fievez, and M. F. Carlier. Continuous monitoring of pi release following nucleotide hydrolysis in actin or tubulin assembly using 2-amino-6-mercapto-7methylpurine ribonucleoside and purine-nucleoside phosphorylase as an enzyme-linked assay. Biochemistry 35:12038-12045, 1996.

${ }^{36}$ Mogilner, A. and L. Edelstein-Keshet. Regulation of actin dynamics in rapidly moving cells: a quantitative analysis. Biophys. J. 83:1237-1258, 2002.

${ }^{37}$ Moriyama, K. and I. Yahara. Two activities of cofilin, severing and accelerating directional depolymerization of actin filaments, are affected differentially by mutations around the actin-binding helix. Embo J. 18:6752-6761, 1999.

${ }^{38}$ Mullins, R., J. Heuser, and T. Pollard. The interaction of arp $2 / 3$ complex with actin: Nucleation, high affinity pointed end capping, and formation of branching networks of filaments. Proc. Natl. Acad. Sci. USA 95:6181-6186, 1998.

${ }^{39}$ Nachmias, V. T., L. Cassimeris, R. Golla, and D. Safer. Thymosin beta 4 (t beta 4 ) in activated platelets. Eur. J. Cell Biol. 61:314-320, 1993.

${ }^{40}$ Ostrander, D. B., J. A. Gorman, and G. M. Carman. Regulation of profilin localization in saccharomyces cerevisiae by phosphoinositide metabolism. J. Biol. Chem. 270:27045-27050, 1995.

${ }^{41}$ Pantiloni, D. and M. Carlier. How profilin promotes actin filament assembly in the presence of thymosin b4. Cell 75:1007-1014, 1993.

${ }^{42}$ Parent, C. A. Making all the right moves: Chemotaxis in neutrophils and dictyostelium. Curr. Opin. Cell Biol. 16:4 13, 2004

${ }^{43}$ Pollard, T.. Rate constants for the reactions of atp-and adp-actin with the ends of actin filaments. J. Cell Biol. 103:2747-2754, 1986.

${ }^{44}$ Pollard, T. D., L. Blanchoin, and R. D. Mullins. Molecular mechanisms controlling actin filament dynamics in nonmuscle cells. Annu. Rev. Biophys. Biomol. Struct. 29:545576, 2000.

${ }^{45}$ Pollard, T. D. and G. G. Borisy. Cellular motility driven by assembly and disassembly of actin filaments. Cell 112:453465, 2003.

${ }^{46}$ Pollard, T. D. and J. A. Cooper. Quantitative analysis of the effect of acanthamoeba profilin on actin filament nucleation and elongation. Biochemistry 23:6631-6641, 1984.

${ }^{47}$ Roos, D., A. A. Voetman, and L. J. Meerhof. Functional activity of enucleated human polymorphonuclear leukocytes. J. Cell Biol. 97:368-377, 1983.
${ }^{48}$ Safer, D., R. Golla, and V. Nachmias. Isolation of a 5kilodalton actin-sequesturing peptide from human blood platelets. Proc. Natl. Acad. Sci. USA 87:2536-2540, 1990.

${ }^{49}$ Selden, L., H. Kinosian, J. Estes, and L. Gershman. Impact of profilin on actin-bound nucleotide exchange and actin polymerization dynamics. Biochemistry 38:2769-2778, 1999.

${ }^{50}$ Suzuki, K., T. Yamaguchi, T. Tanaka, T. Kawanishi, T. Nishimaki-Mogami, K. Yamamoto, T. Tsuji, T. Irimura, T. Hayakawa, and A. Takahashi. Activation induces dephosphorylation of cofilin and its translocation to plasma membranes in neutrophil-like differentiated hl-60 cells. J. Biol. Chem. 270:19551-19556, 1995.

${ }^{5}$ Svitkina, T. M. and G. G. Borisy. Arp2/3 complex and actin depolymerizing factor/cofilin in dendritic organization and treadmilling of actin filament array in lamellipodia. J. Cell Biol. 145:1009-1026, 1999.

${ }^{52}$ Tardy, Y., J. L. McGrath, J. H. Hartwig, and C. F. Dewey. Interpreting photoactivated fluorescence microscopy measurements of steady-state actin dynamics. Biophys. J. 69:1674-1682, 1995.

${ }^{53}$ Theriot, J. and T. Mitchison. Actin microfilament dynamics in locomoting cells. Nature 352:126-131, 1991.

${ }^{54}$ Vallotton, P., S. L. Gupton, C. M. Waterman-Storer, and G. Danuser. Simultaneous mapping of filamentous actin flow and turnover in migrating cells by quantitative fluorescent speckle microscopy. Proc. Natl. Acad. Sci. USA 101:9660-9665, 2004.

${ }^{55}$ Waterman-Storer, C. M., A. Desai, J. C. Bulinski, and E. D. Salmon. Fluorescent speckle microscopy, a method to visualize the dynamics of protein assemblies in living cells. Curr. Biol. 8:1227-1230, 1998.

${ }^{56}$ Wegner, A. Head to tail polymerization of actin. J. Mol. Biol. 108:139-150, 1976.

${ }^{57}$ Weiner, O. D., G. Servant, M. D. Welch, T. J. Mitchison, J. W. Sedat, and H. R. Bourne. Spatial control of actin polymerization during neutrophil chemotaxis. Nat. Cell Biol. 1:75-81, 1999.

${ }^{58}$ Witke, W. The role of profilin complexes in cell motility and other cellular processes. Trends Cell Biol. 14:461-469, 2004.

${ }^{59}$ Yamada, S., D. Wirtz, and S. C. Kuo. Mechanics of living cells measured by laser tracking microrheology (ltm). Biophys. J. 78:1736-1747, 2000. 